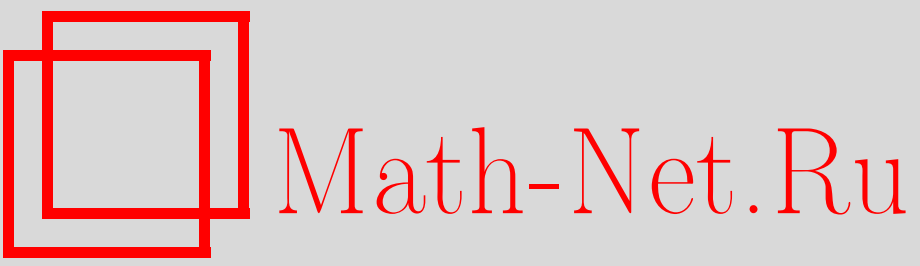

А. Н. Куликов, Бифуркации инвариантных торов у квазилинейных эволюционных уравнений второго порядка в гильбертовом пространстве и сценарий перехода к турбулентности, Итоги науки и техн. Сер. Соврем. мат. и ее прил. Темат. обз., 2019, том 168, 45-52

DOI: https://doi.org/10.36535/0233-6723-2019-168-45-52

Использование Общероссийского математического портала Math-Net.Ru подразумевает, что вы прочитали и согласны с пользовательским соглашением

http: //www.mathnet.ru/rus/agreement

Параметры загрузки:

IP: 54.196 .121 .252

26 апреля 2023 г., 06:58:01 


\title{
БИФУРКАЦИИ ИНВАРИАНТНЫХ ТОРОВ У КВАЗИЛИНЕЙНЫХ ЭВОЛЮЦИОННЫХ УРАВНЕНИЙ ВТОРОГО ПОРЯДКА В ГИЛЬБЕРТОВОМ ПРОСТРАНСТВЕ И СЦЕНАРИЙ ПЕРЕХОДА К ТУРБУЛЕНТНОСТИ
}

\author{
(c) 2019 г. $\quad$ А. Н. КУЛИКОВ
}

\begin{abstract}
АннотАция. В работе рассмотрены квазилинейные дифференциальные уравнения второго порядка в сепарабельном гильбертовом пространстве, для которых может быть реализован известный сценарий Ландау-Хопфа перехода к турбулентности. Показано, что при увеличении управляющего параметра возникают шаг за шагом инвариантные торы, возрастающей размерности. При этом притягивающим оказывается инвариантный тор наибольшей размерности из возможных. Результаты получены при помощи методов качественной теории динамических систем с бесконечномерным пространством начальных условий: метода интегральных многообразий, аппарата теории нормальных форм, а также асимптотических методов анализа динамических систем.
\end{abstract}

Ключевые слова: квазилинейное дифференциальное уравнение, гильбертово пространство, устойчивость, бифуркация, нормальная форма, сценарий Ландау-Хопфа.

\section{BIFURCATIONS OF INVARIANT TORI}

IN SECOND-ORDER QUASILINEAR EVOLUTION

EQUATIONS IN HILBERT SPACES

AND SCENARIOS OF TRANSITION TO TURBULENCE

\author{
(C) 2019 A. N. KULIKOV
}

\begin{abstract}
In this paper, we consider second-order quasilinear differential equations in a separable Hilbert space for which the well-known Landau-Hopf scenario of transition to turbulence can be realized. We prove increasing of the control parameter leads to the consequtive appearance of invariant tori of increasing dimensions. In this case, the invariant torus of the largest possible dimension appears to be attractive. The results are obtained by using methods of the qualitative theory of dynamical systems with an infinite-dimensional space of initial conditions: the method of integral manifolds, the theory of normal forms, and also asymptotic methods of analysis of dynamical systems.

Keywords and phrases: quasilinear differential equation, Hilbert space, stability, bifurcation, normal form, Landau-Hopf scenario.
\end{abstract}

AMS Subject Classification: 37L10, 37L25, 37N15

1. Введение. В основополагающих работах $[11,12,17]$ был предложен сценарий перехода к турбулентности как каскада бифуркаций торов возрастающей размерности. Некоторые примеры реализации такого сценария были предложены в [6-10,12,17]. 
В [7-10] был реализован предложенный Ф. Такенсом план (см. [1]) как каскада бифуркаций в цепочке осцилляторов, связанных только параметрами, в каждом из которых может реализоваться бифуркация Андронова-Хопфа. Ниже будет рассмотрен класс квазилинейных дифференциальных уравнений в полном сепарабельном гильбертовом пространстве. Анализ уравнений из данного класса абстрактных дифференциальных уравнений в гильбертовом пространстве позволяет проследить за цепочкой бифуркаций инвариантных торов и следовательно показать, что сценарий Ландау-Хопфа реализуем. Рассматриваемый класс абстрактных дифференциальных уравнений включает в себя краевые задачи для дифференциальных уравнений с частными производными, имеющие приложения в теории упругой устойчивости. $\mathrm{K}$ таким задачам приводит уравнение, описывающее колебания трубы под воздействием потока протекающей в ней жидкости или газа (см. $[9,10,16])$. Аналогичное уравнение используется при описании колебаний тела вращения в потоке газа (см. $[9,10])$. Близкие задачи возникают при анализе математической модели, известной под названием «мультипликатор-акселератор», если при этом учесть пространственные эффекты (см. [6]).

В настоящей работе абстрактные дифференциальные уравнения исследуются с использованием таких классических методов анализа динамических систем, как метод интегральных (инвариантных) многообразий, аппарат теории нормальных форм Пуанкаре-Дюлака, а также асимптотических методов анализа динамических систем с бесконечномерным фазовым пространством. Центральное место занимает сведение анализа динамики решений рассматриваемых эволюционных уравнений к изучению систем обыкновенных дифференциальных уравнений первого порядка - нормальных форм.

Обоснование результатов базируется на результатах работ $[4,5]$; в первую очередь, речь идет о теореме о сохранении инвариантных торов при возмущениях.

2. Постановка задачи. В действительном полном сепарабельном гильбертовом пространстве $H$ рассмотрим квазилинейное дифференциальное уравнение второго порядка

$$
u_{t t}+2 \varepsilon\left(g_{1} u_{t}+g_{2} A^{2} u_{t}\right)+A^{2} u+2 \varepsilon c B_{1} u_{t}+2 \varepsilon b(c) A u=-2 \varepsilon\left[a_{1}\left(B_{2} u, B_{2} u\right)-a_{2}\left(B_{2} u_{t}, B_{2} u\right)\right] A u,
$$

где $u=u(t)$ - функция со значениями в гильбертовом пространстве, $\varepsilon$ - малый неотрицательный параметр, т.е. $\varepsilon \in\left[0, \varepsilon_{0}\right], 0<\varepsilon_{0} \ll 1, a_{1}, a_{2}, g_{1}, g_{2} \geqslant 0, b=b(c) \in \mathbb{R}$ и зависит от основного управляющего (бифуркационного) параметра $c \geqslant 0, b(0)=0$. Наконец, через $A, A^{2}, B_{1}, B_{2}$ обозначены линейные замкнутые операторы с плотными в $H$ областями определения $H_{A}, H_{A^{2}}$, $H_{B_{1}}, H_{B_{2}}$. Будем считать, то эти линейные операторы (ЛО) обладают следующими свойствами:

1. Оператор $A$ симметричен и положительно определен, т.е. при любых $v_{1}, v_{2} \in H_{A}$ справедливо равенство $\left(A v_{1}, v_{2}\right)=\left(v_{1}, A v_{2}\right)$ и неравенство $\left(A v_{1}, v_{1}\right) \geqslant \gamma_{0}^{2}\left(v_{1}, v_{1}\right)$, где $\gamma_{0}>0$, а через $(\cdot, \cdot)$ обозначено скалярное произведение в $H$.

2. Оператор $A$ имеет счетный набор простых положительных собственных значений $\sigma_{1}<\sigma_{2}<$ $\ldots<\sigma_{n}<\ldots$ Им соответствуют собственные элементы $h_{j}, j=1,2, \ldots$. Будем считать, что семейство собственных элементов образует полную ортонормированную систему в сепарабельном гильбертовом пространстве $H$. В частности, $\left(h_{j}, h_{k}\right)=0, j \neq k ;\left(h_{k}, h_{k}\right)=1$.

3. 3. Оператор $i A$ является производящим оператором группы линейных ограниченных операторов в комплексном расширении $H\left(w \in H_{c}\right.$, если $\left.w=u+i v, u, v \in H\right)$.

4. 4. Операторы $B_{1}, B_{2}$ подчинены оператору $A$ и, следовательно, $H_{A} \subseteq H_{B_{1}}, H_{B_{2}}\left(H_{A^{2}} \subseteq H_{A}\right)$.

5. 5. Семейство элементов $q_{k}=B_{2} h_{k}$ образует ортогональную систему в $H$.

Сформулированные предложения носят характер общности положений. Их наличие гарантируют общие свойства решений уравнения (1). Если уравнение (1) дополнить начальными условиями

$$
u(0)=u_{0} \in H_{A^{2}}, \quad u_{t}(0)=\dot{u}_{0} \in H_{A},
$$

то при достаточно малой величине $\varepsilon_{0}\left(\varepsilon \in\left[0, \varepsilon_{0}\right]\right)$ задача Коши корректно разрешима на отрезке $\left[-T\left(\varepsilon_{0}\right), T\left(\varepsilon_{0}\right)\right], T\left(\varepsilon_{0}\right)>0$, и $\lim _{\varepsilon_{0} \rightarrow 0} T\left(\varepsilon_{0}\right)=\infty$, если $\left\|u_{0}\right\|_{H_{A^{2}}} \leqslant r_{0},\left\|\dot{u}_{0}\right\|_{H_{A}} \leqslant r_{0}$, где $r_{0}$ - некоторая положительная постоянная. При этом, конечно, $T\left(\varepsilon_{0}\right)=T\left(\varepsilon_{0}, r_{0}\right)$, т.е., вообще говоря, зависит от $r_{0}$. Справедливость последнего замечания вытекает из результатов работ $[14,15]$. 
Замечание 1. Некоторые из предположений 1-5 могут быть изменены и даже усилены, но такой вариант условий включает в себя многие прикладные задачи теории аэрогидроупругости, а также макроэкономики (см., например, [3]). Некоторые более актуальные предположения будут сделаны позже, в конце следующего раздела данной работы.

При $\varepsilon=0$ получаем линейное дифференциальное уравнение в $H$

$$
u_{t t}+A^{2} u=0
$$

которое имеет счетный набор решений в комплексной форме $w_{k}=\exp \left(i \sigma_{k} t\right)$, а также $\bar{w}_{k}(t)$. Базисные решения можно записать и в действительной форме: $h_{k} \cos \sigma_{k} t, h_{k} \sin \sigma_{k} t$. Задача Коши (3), (2) имеет решение

$$
u(t)=\sum_{k=1}^{\infty}\left[p_{k} \cos \sigma_{k} t+\frac{q_{k}}{\sigma_{k}} \sin \sigma_{k} t\right] h_{k},
$$

где $p_{k}=\left(u_{0}, h_{k}\right), q_{k}=\left(\dot{u}_{0}, h_{k}\right)$. В частности, в норме $H$ сходятся ряды

$$
\sum_{k=1}^{\infty} p_{k} \sigma_{k}^{2} h_{k}, \quad \sum_{k=1}^{\infty} q_{k} \sigma_{k} h_{k}
$$

а также числовые ряды

$$
\sum_{k=1}^{\infty} p_{k}^{2} \sigma_{k}^{4}, \quad \sum_{k=1}^{\infty} q_{k}^{2} \sigma_{k}^{2}
$$

Сходимость последних двух рядов является следствием полноты ортонормированной системы собственных элементов.

В следующих разделах будут рассмотрены вопросы, связанные с поведением решений уравнения (1) из достаточно малой окрестности нулевого решения, точнее? с начальными условиями $\left\|u_{0}\right\|_{A^{2}} \leqslant r_{0},\left\|\dot{u}_{0}\right\|_{A} \leqslant r_{0}$, где $r_{0}>0$; обычно эта постоянная достаточно мала. Наконец,

$$
\|u\|_{A}=\|u\|+\|A u\|, u \in H_{A}, \quad\|u\|_{A^{2}}=\|u\|+\|A u\|+\left\|A^{2} u\right\|, u \in H_{A},
$$

где $\|u\|=\sqrt{(u, u)}$.

3. Нормальная форма. Решение дифференциального уравнения (1) с начальными условиями из достаточно малой окрестности нулевого решения (из достаточно малого шара с центром в нуле пространства $E=H_{A^{2}} \times H_{A}$ - фазового пространства решений дифференциального уравнения (1)) будем искать в следующем виде:

$$
u(t, \varepsilon)=w(t, z)+\varepsilon v(t, z, \varepsilon), \quad w=w(t, z)=\sum_{k=1}^{\infty}\left[z_{k} \exp \left(i \sigma_{k} t\right)+\bar{z}_{k} \exp \left(-i \sigma_{k} t\right)\right] h_{k},
$$

где $z_{k}=z_{k}(t), z=z(t)=\left\{z_{1}(t), z_{2}(t), z_{3}(t), \ldots\right\}$ - счетный набор комплекснозначных функций переменного $t$. Далее, будем предполагать, что априори при всех рассматриваемых значениях $t$ бесконечномерный вектор $z(t) \in l_{2,2}$. Напомним, что бесконечномерный вектор $a=\left(a_{1}, a_{2}, a_{3}, \ldots\right) \in$ $l_{2}$, если сходится ряд $\sum_{j=1}^{\infty}\left|a_{j}\right|^{2}, a \in l_{2,2}$, если сходится ряд $\sum_{j=1}^{\infty} \sigma_{j}^{4}\left|a_{j}\right|^{2}$; здесь $a_{j} \in C$. В иной терминологии, запись $a \in l_{2}$ означает, что $a$ принадлежит дискретному аналогу $H_{c}$, а $a \in l_{2,2}-$ что $a$ принадлежит дискретному аналогу пространства $H_{c A^{2}}$. Последние предположения эквивалентны включению функции $w(t)$ в фазовое пространство решений уравнения $(1)$ т.е. $w(t) \in H_{A^{2}}$ и $w_{t}(t) \in H_{A}$. В свою очередь, функцию $w(t)$ выбираем таким образом, чтобы функции $z_{k}(t)$ из последовательности $z(t)=\left\{z_{1}(t), z_{2}(t), \ldots\right\}$ удовлетворяли бесконечной системе обыкновенных дифференциальных уравнений

$$
\dot{z}_{k}=\varepsilon \varphi_{k}(z, \varepsilon), \quad k=1,2, \ldots
$$


Правые части системы (9) будут выбраны далее в процессе реализации алгоритма построения системы уравнений (9), которую принято называть нормальной формой. При этом основное внимание следует уделить не построению правых частей системы (9), а «укороченной» системе

$$
\dot{z}_{k}(t)=\varepsilon f_{k}(z)
$$

где $f_{k}(z)=\left.\varphi_{k}(z, \varepsilon)\right|_{\varepsilon=0}$. Систему дифференциальных уравнений (10) принято называть укороченной нормальной формой (УНФ; в англоязычной литературе - truncated normal form, TNF).

Главная часть в равенстве (8) обозначена через $w(t)$; эта абстрактная функция, записанная в виде ряда (8), является квазипериодической функцией с частотами $\sigma_{1}, \ldots, \sigma_{n}, \ldots$ (во многих случаях из приложений из теории упругой устойчивости она оказывается даже периодической с периодом $\left.2 \pi / \sigma_{1}\right)$. Далее будем считать, что набор частот $\sigma_{1}, \sigma_{2}, \ldots$ обладает следующими свойствами:

(1) при $k<m$ справедливо неравенство $\left|2 \sigma_{k}-\sigma_{m}\right| \geqslant M_{0}$, где $M_{0}$ - некоторая положительная постоянная;

(2) при $k \geqslant m$ справедливо неравенство $\left|2 \sigma_{k}-\sigma_{m}\right| \geqslant \sigma_{k}$.

Последние два свойства собственных значений в подавляющем числе примеров реализуются и проверяются достаточно элементарно. Так, например, если $A v=-v^{\prime \prime}(x), v(0)=v(\pi)=0$, то $\sigma_{k}=k^{2}$, и поэтому эти свойства очевидны. В частности, в качестве $M_{0}$ можно выбрать 1.

Приступим к изложению способа вычисления правых частей системы (10). Для этого обозначим через $y(t, z)$ главную часть функции $v(t, z, \varepsilon)$, т.е. положим $y(t, z)=v(t, z, 0)$.

Подставим теперь сумму (8) в уравнение (1), вычислим производные функций в силу системы (10) и выделим слагаемые при $\varepsilon$. В результате получим неоднородное уравнение для определения $y(t, z)$, при анализе которого интерпретируем $z=\left(z_{1}, z_{2}, z_{3}, \ldots\right)$ как параметры.

Итак, для $y(t, z)$ получаем линейное неоднородное дифференциальное уравнение

$$
y_{t t}-A^{2} y=F(t, z),
$$

где правая часть уравнения (11) определена следующим равенством:

$$
\begin{gathered}
F(t, z)=-2 \sum_{k=1}^{\infty}\left[f_{k} i \sigma_{k} \exp \left(i \sigma_{k} t\right)-\bar{f}_{k} i \sigma_{k} \exp \left(-i \sigma_{k} t\right)\right] h_{k}- \\
-2 \sum_{k=1}^{\infty}\left[\left(g_{1}+g_{2} \sigma_{k}^{2}\right) i \sigma_{k} z_{k} \exp \left(i \sigma_{k} t\right)-\left(g_{1}+g_{2} \sigma_{k}^{2}\right) i \sigma_{k} \bar{z}_{k} \exp \left(-i \sigma_{k} t\right)\right] h_{k}- \\
-2 c \sum_{k=1}^{\infty}\left[i \sigma_{k} z_{k} \exp \left(i \sigma_{k} t\right)-i \sigma_{k} \bar{z}_{k} \exp \left(-i \sigma_{k} t\right)\right] B_{1} h_{k}-2 b \sum_{k=1}^{\infty}\left[z_{k} \exp \left(i \sigma_{k} t\right)+\bar{z}_{k} \exp \left(-i \sigma_{k} t\right)\right] \sigma_{k} h_{k}- \\
-2 b \sum_{k=1}^{\infty}\left[z_{k} \exp \left(i \sigma_{k} t\right)+\bar{z}_{k} \exp \left(-i \sigma_{k} t\right)\right] \sigma_{k} h_{k} \Phi,
\end{gathered}
$$

где

$$
\Phi=a_{1}\left(B_{2} w, B_{2} w\right)+a_{2}\left(B_{2} w_{t}, B_{2} w\right)
$$

и, следовательно,

$$
\begin{gathered}
\Phi=a_{1} \sum_{k=1}^{\infty}\left(z_{k} \exp \left(i \sigma_{k} t\right)+\bar{z}_{k} \exp \left(-i \sigma_{k} t\right)\right)^{2} \omega_{k}^{2}+a_{2} \sum_{k=1}^{\infty}\left(i \sigma_{k} z_{k} \exp \left(i \sigma_{k} t\right)-i \sigma_{k} \bar{z}_{k} \exp \left(-i \sigma_{k} t\right)\right) \omega_{k}^{2}, \\
\omega_{k}^{2}=\left(B_{2} h_{k}, B_{2} h_{k}\right)=\left(q_{k}, q_{k}\right) .
\end{gathered}
$$

Напомним, что уравнение (11) разрешимо в классе квазипериодических функций с базисом частот $\sigma_{1}, \sigma_{2}, \ldots$, если выполнены следующие равенства:

$$
\lim _{T \rightarrow \infty} \frac{1}{2 T} \int_{-T}^{T}\left(F, h_{k}\right) \exp \left( \pm i \sigma_{k} t\right) d t=0
$$


где $k=1,2,3, \ldots$ (см., например, [2]). Относительно простые вычисления показывают, что

$$
f_{k}(z)=-\left(g_{1}+g_{2} \sigma_{k}^{2}\right) z_{k}+c \nu_{k} z_{k}-a_{2} \sigma_{k} \omega_{k}^{2} z_{k}\left|z_{k}\right|^{2}+i a_{1} z_{k} \sum_{m=1}^{\infty} \omega_{m}^{2}\left|z_{m}\right|^{2} .
$$

Добавим, что линейный оператор $B_{2}$ подчинен $A\left(\left\|B_{2} v\right\| \leqslant M_{1}\|A u\|, u \in H_{A}, M_{1}>0\right)$, и поэтому ряд $\sum_{m=1}^{\infty} \omega_{m}^{2}\left|z_{m}\right|^{2}$ сходится, если сходится ряд $\sum_{m=1}^{\infty} \sigma_{m}^{2}\left|z_{m}\right|^{2} ;$ более того, $\omega_{m}^{2} \leqslant M_{1}^{2} \sigma_{m}^{2}$. Наконец, использовано обозначение $\nu_{k}=\left(B_{1} h_{k}, h_{k}\right)$.

Следовательно, укороченную нормальную форму (10) можно записать в виде

$$
\dot{z}_{k}=\varepsilon\left[-\left(g_{1}+g_{2} \sigma_{k}^{2}\right) z_{k}+c \nu_{k} z_{k}+i b(c) z_{k}-a_{2} \sigma_{k} \omega_{k}^{2}\left|z_{k}\right|^{2} z_{k}+i a_{1} \sigma_{k} z_{k} \sum_{m=1}^{\infty} \omega_{m}^{2}\left|z_{m}\right|^{2}\right],
$$

где $k=1,2,3, \ldots$ Ненулевые решения системы дифференциальных уравнений (13) удобно искать в следующем виде:

$$
z_{k}(t)=\rho_{k}(t) \exp \left(i \varphi_{k}(t)\right)
$$

где $\rho_{k}^{2}(t)=\left|z_{k}(t)\right|^{2}, \varphi_{k}(t)$ - аргумент комплекснозначной функции $z(t)$.

В результате получаем систему действительных дифференциальных уравнений

$$
\begin{aligned}
\dot{\rho}_{k} & =\varepsilon \rho_{k}\left[\beta_{k}-d_{k} \rho_{k}^{2}\right], \\
\dot{\varphi}_{k} & =\varepsilon\left[b(c) \sigma_{k}+\sigma_{k} a_{1} \sum_{m=1}^{\infty} \omega_{m}^{2} \rho_{m}^{2}\right],
\end{aligned}
$$

где

$$
\beta_{k}=\beta_{k}(c)=c \nu_{k}-\left(g_{1}+g_{2} \sigma_{k}^{2}\right), \quad \nu_{k}=\left(B_{1} h_{k}, h_{k}\right), \quad d_{k}=a_{2} \sigma_{k} \omega_{k}^{2}>0 .
$$

Далее будем считать, что $\nu_{k}>0$; последовательность $\left\{c_{k}\right\}$, где $c_{k}=\left(g_{1}+g_{2} \sigma_{k}^{2}\right) / \nu_{k}, k=1,2,3, \ldots$, монотонно возрастает; $\lim _{k \rightarrow \infty} c_{k}=\infty$. Отметим, что $\beta_{k}(0)<0$ при всех натуральных $k$.

Из этих предположений вытекает, что можно указать $c=c_{m}$, при котором

$$
\begin{array}{ll}
\text { (1) } \beta_{m}\left(c_{m}\right)=0 ; & \\
\text { (2) } \beta_{k}\left(c_{m}\right)>0, & \text { если } k<m \text {; } \\
\text { (3) } \beta_{p}\left(c_{m}\right)<0, & \text { если } p>m .
\end{array}
$$

Следовательно, при $c \in I_{m}=\left(c_{m}, c_{m+1}\right]$ выполнены неравенства $\beta_{k}(c)>0$, если $k \leqslant m$, но $\beta_{p}(c) \leqslant 0$ при $p>m$.

При таком выборе $c, c \in I_{m}$, получаем, что

(1) $\lim _{t \rightarrow \infty} \rho_{s}(t)=0, \quad$ если $s=m+1, m+2, m+3, \ldots, t \rightarrow \infty$;

(2) $\lim _{t \rightarrow \infty} \rho_{k}(t)=\alpha_{k}, \quad \alpha_{k}=\sqrt{\beta_{k}(c) / a_{2} \sigma_{k} \omega_{k}^{2}}-$ состояние равновесия уравнения с номером $k \leqslant m$.

Отсюда вытекает, что система дифференциальных уравнений (15) при $c \in I_{m}$ имеет $2^{m}$ состояний равновесия $S_{l, m}=\left(\eta_{1}, \ldots, \eta_{m} ; 0, \ldots, 0, \ldots\right)$; первые $m$ компонент могут быть ненулевыми, а $\rho_{j}=0$, если $j \geqslant m+1$. Итак, коэффициенты $\eta_{j}, j=1, \ldots, m$, может быть равна либо $\alpha_{j}$, либо 0 ; при этом число $l$ указывает на число ненулевых компонент у $S_{l, m}$. Так, $S_{0, m}$ - нулевое состояние равновесия, $S_{1, m}$ имеет одну ненулевую компоненту среди координат вектора $\eta=\left(\eta_{1}, \eta_{2}, \ldots, \eta_{m}\right) \in \mathbb{R}^{m}$. Особую роль играет состояние равновесия $S_{m, m}$, у которого все первые $m$ координат положительны $\left(\eta_{m}=\alpha_{m}>0\right)$.

Пусть $G_{k}(\rho, c)=\rho_{k}\left[\beta_{k}(c)-d_{k} \rho^{2}\right]$. Разделим систему дифференциальных уравнений (15) на две подсистемы. Первая состоит из первых $m$ уравнений системы $(15)$ :

$$
\dot{\rho}_{k}=\varepsilon G_{k}\left(\rho_{k}, c\right), \quad k=1,2, \ldots, m,
$$

а вторая - из остальных:

$$
\dot{\rho}_{s}=\varepsilon G_{k}\left(\rho_{s}, c\right), \quad s=m+1, m+2, \ldots
$$


Для уравнений системы (18) характерно выполнение неравенств $\dot{\rho}_{s}<0$ и,следовательно, в этом случае $\lim _{t \rightarrow \infty} \rho_{s}=0$. Вопрос об устойчивости любого состояния вида $S_{l, m}$ сводится к анализу спектра матрицы Якоби системы (17), вычисленной для соответствующего состояния равновесия. Очевидно, она диагональная, т.е.

$$
J=\operatorname{diag}\left\{\lambda_{j}(c)\right\}
$$

где

а $\rho_{k}=\alpha_{k}$ или $\rho_{k}=0$. При этом

$$
\lambda_{j}(c)=\left.G_{k}^{\prime}\left(\rho_{k_{j}}(c)\right)\right|_{\rho_{k}=\eta},
$$

$$
\left.G_{k}^{\prime}\left(\rho_{k}, c\right)\right|_{\rho_{k}=0}=\beta_{k}>0,\left.\quad G_{k}^{\prime}\left(\rho_{k}, c\right)\right|_{\rho_{k}=\alpha_{k}}=-2 \beta_{k}<0 .
$$

Отсюда вытекает справедливость следующего утверждения.

Лемма 1. Пусть $c \in I_{m}$. Тогда система дифференциальных уравнений (15) имеет $2^{m}$ состояний равновесия $S_{l, m}$. Все они неустойчивы, за исключением одного, а именно, $S_{m, m}$, т.е. состояния равновесия с максимально возможным числом положительных компонент $\rho_{k}=\alpha_{k}$, $k=1,2, \ldots, m$.

Перейдем теперь к системе (16). Состоянию равновесия $S_{l, k}=\left(\eta_{1}, \ldots, \eta_{m} ; 0,0, \ldots\right)$, где $\eta_{k_{j}}=$ $\alpha_{k_{j}}$, а остальные компоненты вектора $\eta=\left(\eta_{1}, \ldots, \eta_{m}\right)$ равны нулю, соответствуют решения системы (16) вида

$$
\Phi_{k_{j}}(t)=\varepsilon \Theta_{k_{j}} t+\varphi_{k_{j} 0},
$$

где $\Theta_{k_{j}}=\left(b+a_{1} \sum_{j=1}^{l} \omega_{k_{j}}^{2} \alpha_{k_{j}}^{2}\right) \sigma_{k_{j}}, \varphi_{k_{j}, 0}$ - произвольные действительные постоянные.

Состоянию равновесия $S_{l, m}$ системы обыкновенных дифференциальных уравнений (16) соответствуют квазипериодические решения нормальной формы (13)

$$
z_{k_{j}}(t)=\alpha_{k_{j}}\left(i \Theta_{k_{j}} \varepsilon t+i \varphi_{k_{j}, 0}\right), \quad z_{p}=0, q \quad p \neq k_{j} .
$$

Решение может быть периодическим, если $\theta_{k_{j}}$ соизмеримы.

Семейство решений (21) образует инвариантный тор $T_{T N F, l}(\varepsilon)$ размерности $l$. Все такие торы седловые, если $l<m$. При $l=m$ имеем асимптотически устойчивый (притягивающий) тор $T_{T N F, m}(\varepsilon)$ размерности $m$.

Из предшествующих построений и результатов работ [4,5] вытекает, что справедливо следующее утверждение.

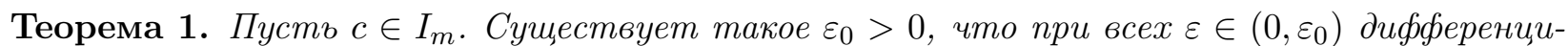
альное уравнение (1) имеет инвариантный тор $T_{l}(\varepsilon)$ размерности $l$, соответствующий инвариантному тору $T_{T N F, l}(\varepsilon)$ нормальной формы (13) (состоянию равновесия $S_{l, m}$ системы обыкновенных дифференциальных уравнений (15)). При $l<m$ торы $T_{l}(\varepsilon)-$ седловые, а тор $T_{m}(\varepsilon)-$ локальный аттрактор для решений дифференциального уравнения (1). Для решений на $T_{l}(\varepsilon)$ справедлива асимптотическая формула

$$
u(t, \varepsilon)=2 \sum_{j=1}^{m} \rho_{k_{j}} \cos \left(\sigma_{k_{j}} t+\varphi_{k_{j} 0}\right)+O(\varepsilon)
$$

где $\rho_{k_{j}}=\alpha_{k_{j}}$, т.е. набору положительных координат состояния равновесия системы дифференциальных уравнений.

4. Сценарий Ландау-Хопфа. Пусть сначала $c \in I_{m}=\left(c_{m}, c_{m+1}\right]$. Тогда уравнение (1) имеет набор из $2^{m}-1$ инвариантных торов $T_{l}(\varepsilon)$ размерности $l=1,2, \ldots, m$ (тор нулевой размерности $T_{0}(\varepsilon)$ - это нулевое состояние равновесия). При этом, как уже отмечалось, будет лишь один тор $T_{m}(\varepsilon)$ максимальной размерности при данном диапазоне изменения $c$.

Увеличим теперь $c$ таким образом, что $c$ перейдет из $I_{m}$ в $I_{m+1}=\left(c_{m+1}, c_{m+2}\right]$. Тогда уравнение (1) будет иметь уже $l=2^{m+1}$ инвариантных торов $T_{l}(\varepsilon)$. При этом торы, существующие при $c \subset I_{m}$, сохраняются, но добавляются еще $2^{m}$ инвариантных торов, среди которых $T_{m+1}(\varepsilon)$; 
именно он теперь стал устойчивым. Данный тор возник в результате бифуркации тора $T_{m}(\varepsilon)$ максимально возможной размерности при $c \in I_{m}$.

Следует отметить, что при увеличении $c$ можно получить тор достаточно большой размерности $N=N(\varepsilon)$, но предельный период, естественно, невозможен, так как в такой интерпретации $c \rightarrow+\infty$ и, принципе, рассмотреть «предельное» уравнение не представляется возможным.

Приведем пример краевой задачи, которая может быть включена в рассматриваемый класс абстрактных уравнений. Рассмотрим уравнение с частными производными

$$
\begin{aligned}
u_{t t}+2 \varepsilon\left[g_{1} u_{t}+g_{2} u_{t x x x x}\right]+u_{x x x x}+\varepsilon c^{2} u_{x x}+\varepsilon \beta c u_{t x} & +\varepsilon \alpha c^{2} u_{x}= \\
& =\varepsilon u_{x x}\left[2 a_{1} \int_{0}^{\pi}\left(u_{x}\right)^{2} d x+2 a_{2} \int_{0}^{\pi} u_{x} u_{t x} d x\right],
\end{aligned}
$$

где функция $u(t, x)$ удовлетворяет краевым условиям

$$
u(t, 0)=u(t, \pi)=u_{x x}(t, 0)=u_{x x}(t, \pi)=0 .
$$

Краевая задача (23), (24) приведена в нормированном виде; она описывает колебания трубы под воздействием потока жидкости, протекающей в ней со скоростью $c$ (нормированная величина), в поле силы тяжести (см., например, [16]). В дифференциальном уравнении (23) коэффициенты $c, a_{1}, a_{2}, g_{1}, g_{2} \in \mathbb{R}_{+}, \alpha, \beta \in \mathbb{R} ;$ наконец, $u(t, x)$ - нормированное отклонение осевой линии трубы от тривиального состояния равновесия. В уравнении (23) присутствует малый параметр $\varepsilon>0$. При стандартной перенормировке $\varepsilon \approx E^{-1 / 2}$, где $E$-модуль упругости материала, из которого изготовлена труба (для стали $E \approx 2 \cdot 10^{11} \mathrm{H} / \mathrm{M}^{2}$, т.е. величина $E^{-1 / 2} \sim 10^{-5}$ может быть интерпретирована как малый параметр).

В примере роль $A$ играет дифференциальный оператор

$$
A v=-v^{\prime \prime}, \quad \text { где } \quad v=v(x), \quad v(0)=v(\pi)=0 .
$$

Поэтому в качестве $H_{A}$ можно выбрать пространство $\stackrel{\circ}{W} \underset{2}{2}(0, \pi)$ - подпространство функций из $W_{2}^{2}[0, \pi]$, удовлетворяющих краевым условиям $v(0)=v(\pi)=0$. Наконец, $W_{2}^{p}[0, \pi]-$ стандартное обозначение для пространств Соболева (см. [13]).

\section{СПИСОК ЛИТЕРАТУРЫ}

1. Брур Х. В., Дюмортье Ф., ван Стрин С., Такенс Ф. Структуры в динамике. - М.-Ижевск: Ин-т комп. исслед., 2003.

2. Демидович Б. П. Лекции по математической теории устойчивости. - М.: Наука, 1967.

3. Кокуйкин $E$. C., Куликов A. H. Циклы и торы деловой активности в одной математической модели макроэкономики// Модел. анал. информ. систем. - 2009. - 16, № 4. - С. 86-95.

4. Колесов А. Ю., Куликов А. Н., Розов Н. Х. Инвариантные торы одного класса точечных отображений: принцип кольца// Диффер. уравн. - 2003. - 39, № 5. - С. 584-601.

5. Колесов А. Ю., Куликов А. Н., Розов Н. Х. Инвариантные торы одного класса точечных отображений: сохранение тора при возмущениях// Диффер. уравн. - 2003. - 39, № 6. - С. 738-753.

6. Колесов А. Ю., Куликов А. Н., Розов H. Х. Развитие турбулентности по Ландау в модели «мультипликатор-аксельратор»// Докл. РАН. - 2008. - 42, № 6. - С. 739-743.

7. Куликов A. Н. Аттракторы двух краевых задач для модифицированного телеграфного уравнения// Нелин. динам. - 2008. - 4, № 1. - С. 56-67.

8. Куликов А. Н. Возможность реализации сценария Ландау перехода к турбулентности в задаче о колебаниях трубы, транспортирующей жидкость// в кн.: Тр. VIII Всеросс. конф. «Нелинейные колебания механических систем». - Н. Новгород, 2008. - 2. - С. 378-380.

9. Куликов А. Н. О возможности реализации сценария Ландау-Хопфа перехода к турбулентности в двух задачах теории упругой устойчивости// Диффер. уравн. - 2011. - 47, № 2. - С. 296-298.

10. Куликов A. Н. О реализации сценария Ландау-Хопфа перехода к турбулентности в некоторых задачах теории упругой устойчивости// Диффер. уравн. - 2012. - 48, № 9. - С. 1278-1291.

11. Ландау Л. Д. К проблемам турбулентности// Докл. АН СССР. - 1944. - 44, № 8. - С. 339-342. 
12. Ландау Л. Д., Лифиии Е. М. Теоретическая физика. Т. 6. Гидродинамика. - М.: Наука, 1988.

13. Соболев C. Л. Некоторые применения функционального анализа в математической физике. - Изд-во Ленингр. ун-та, 1950.

14. Соболевский П. Е. Об уравнениях параболического типа в банаховом пространстве// Тр. Моск. мат. о-ва. - 1967. - 10. - С. 297-350.

15. Якубов С. Я. Разрешимость задачи Коши для абстрактных квазилинейных гиперболических уравнений второго порядка и их приложениях// Тр. Моск. мат. о-ва. - 1970. - 22. - С. 37-60.

16. Holmes P. J., Marsden J. E. Bifurcation of dynamical systems and nonlinear oscillations in engineering systmes// in: Lect. Notes Math.. — Berlin: Springer-Verlag, 1978. - 648. — P. 165-206.

17. Hopf E. A mathematical example displaying the features of turbulence// Commun. Pure Appl. Math. 1948. — № 1. - P. 303-322.

Куликов Анатолий Николаевич

Ярославский государственный университет им. П. Г. Демидова

E-mail: anat_kulikov@mail.ru 